\title{
AUTOMATIC CREATION OF HYPNOGRAM
}

Polysomnography is an important diagnostic method used in sleep monitoring of patients with various sleep disorders. The formulation of a precise diagnosis requires correct evaluation and interpretation of polysomnography EEG and this evaluation is provided by a doctor. In order to save time, various automatic evaluations methods have been developed. One of these methods is based on signal division into 30 second long sections which are subsequently analyzed and reduced using adaptive segmentation and parameters are computed from these segments. Cluster analysis is used to divide individual segments and the class with biggest quantity of segments is called "priority class". Definition of sleep states is then based on priority class. The end result is a hypnogram and the average success rate of the proposed method is about 68\%.

\section{Sleep}

Sleep is a naturally occurring state of relatively suspended sensory and motor activity, characterized by total or partial unconsciousness and the inactivity of nearly all voluntary muscles. It is distinguished from quiet wakefulness by a decreased ability to react to stimuli. Various sleep stages are differentiated during sleep. Sleep architecture is made of sleep states.

The following sleep states exist:

- NREM - non-rapid eye movement sleep, there are three subtypes: NREM1, NREM2, NREM3-4,

- REM - rapid eye movement sleep.

Typical young human sleep is created by 4 or 6 , alternating, 90 minute long NREM and REM sleep loops. The number of deep sleep and REM sleep stages decreases with age, by contrast increasing the number of short wakes. [1].

\section{Diagnostics}

Special laboratories for the study of sleep states exist. Various measurement methods are used in the process of sleep analysis. One such method is called polysomnography (PSG), which is an important diagnostic method for sleep monitoring of patients with sleep disorders or other psychical defects. The following biosignals and quantities are measured: EEG, EMG, ECG, EOG, respiration and blood oxygen saturation).

The resulting polysomnography record has a length of 8 hours and is subsequently divided into 30 second long sites which are then analyzed. However, the actual evaluation of sleep activity is very time-consuming and is provided by a doctor. In order to save time, various automatic evaluations methods are developed and one such method is detailed in the following paragraphs.

\section{Implementation of the Automatic Sleep State Detection Algorithm}

The proposed algorithm was tested on PSG records of children and consists of several steps. In our case we only used EEG and EOG records from all signals recorded in PSG. The total length of all records was approximately 70 hours and the target group consisted of children aged 6-15 years.

The main body of the algorithm is based on these basic steps:

- Creation of epochs

- Adaptive segmentation

- Cluster analysis

- Sleep state definition

- Hypnogram

Creation of epoch and adaptive segmentation

Several hours long EEG record (6-8 hours) is divided into 30 second long sites - epochs.

EEG signal is stochastic and non-stationary. Automatic analysis of EEG signal using computer equipment is primarily focused on the extraction of informative features with maximum possible discriminatory ability. By obtaining the spectral appearance of part of a signal with constant length one can also obtain signal deformation characteristics.

Adaptive segmentation is necessary because the EEG signal is non-stationary. Therefore, epochs are processed using adaptive seg-

\footnotetext{
* M. Gala ${ }^{1}$, B.Babusiak ${ }^{1}$, V. Novak $^{2}$

${ }^{1}$ Department of Electromagnetic and Biomedical Engineering, Faculty of Electrical Engineering, University of Zilina, Slovakia,

E-mail: michal.gala@fel.uniza.sk

${ }^{2}$ Children's neurology clinic, University Hospital Ostrava, Ostrava-Poruba, Czech Republic
} 


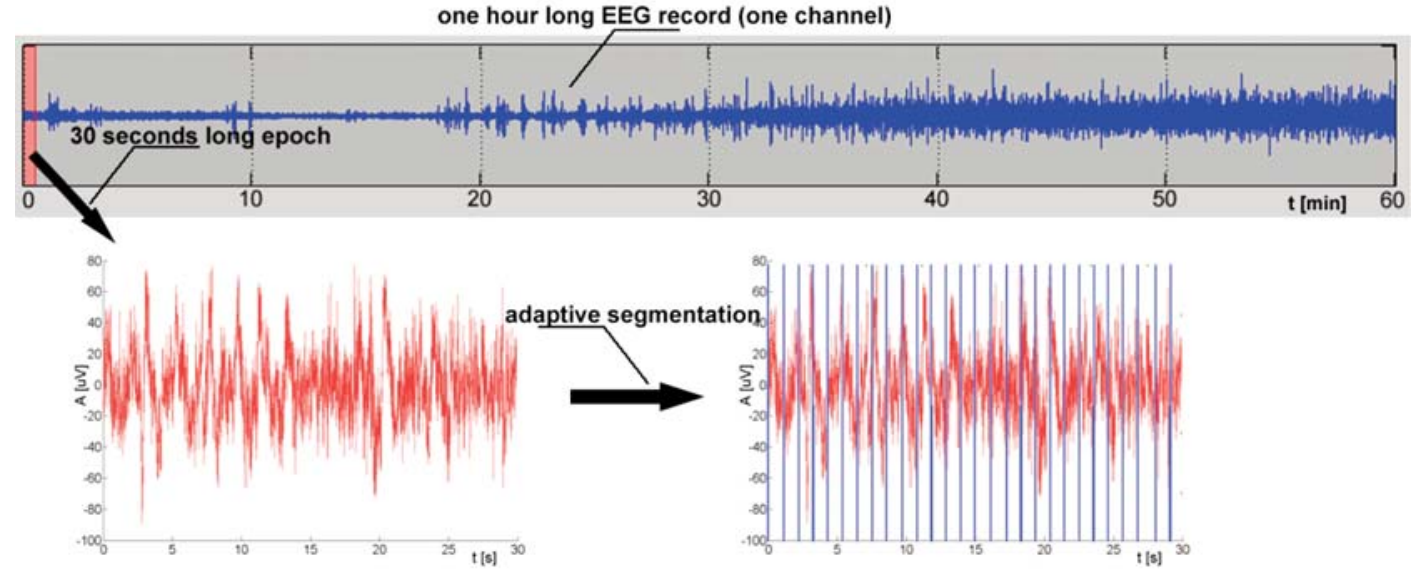

Fig. 1 Creation of 'epochs' and adaptive segmentation

mentation [4] and in our implementation a method based on signal standard deviation is applied.

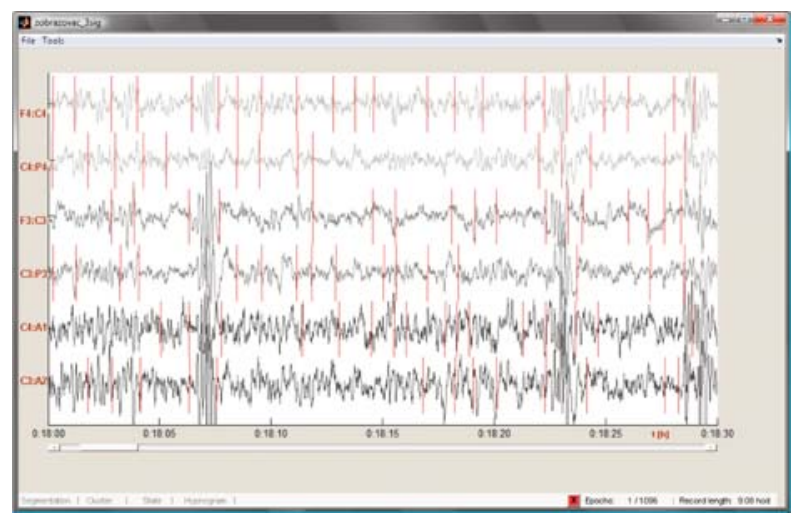

Fig. 2 Application of adaptive segmentation on EEG signal

\section{Cluster analysis}

In this step the generated segments are divided into classes using cluster analysis. Cluster analysis is the classification of objects into different groups, or more precisely, the partitioning of a data set into subsets (clusters), so that the data in each subset share some common traits - often proximity according to certain defined distance measure. Data clustering is a common technique for statistical data analysis, which is used in many applications (including data mining, pattern recognition, image analysis, bioinformatics and many more).

One of the simplest, non-hierarchical clustering methods is the K-means clustering method. The K-means algorithm assigns each point to the cluster whose center is the nearest. The center is the average of all the points in the cluster. Its coordinates are the arithmetic mean for each dimension separately over all the points in the cluster. The main advantages of this algorithm are its simplicity and speed which allows it to run on large datasets [5, 6]. In our case the cluster analysis splits segments into five different classes which were chosen empirically. Segments are then divided into classes based on specific parameters.

\section{Parameter computation}

Certain specific characteristics are required in order to best describe the electroencephalograph segments. Such characteristics include the time and frequency domain of EEG segments [4], amplitude variability, difference between maximum positive and minimum negative value of amplitude, $1^{\text {st }}$ and $2^{\text {nd }}$ derivation maximum value, average frequency and Hjorth parameters.

\section{Sleep state definition and hypnogram}

Segments are divided into classes for each of six channels independently. This means that, for example, class no. 1 can have different parameters in channel 1 compared to the same class in channel 2. In the next step the total number of segments in all classes is detected. If one of the classes has more than $66 \%$ of all segments (for one channel), this class is marked a priority class.

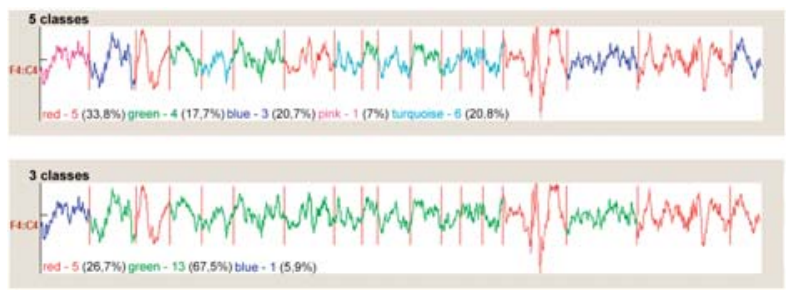

Fig. 3 Priority class definition

The actual sleep state is defined based on the priority class. If a priority class does not exist, cluster analysis with smaller number of classes is used. A simple neural network was used for the sleep state definition [2]. Six epochs of each sleep state (awake, REM, NREM1, NREM2, NREM3/4) were chosen for neural network training. All epochs (from channel F4:C4, C4:P4, F3:C3, C3:P3, $\mathrm{C} 4: \mathrm{A} 1, \mathrm{C} 3: \mathrm{A} 2)$ were preprocessed by adaptive segmentation and all segments were described by Hjorth's parameters. These para- 
meters were then used as a training set for the neural network [3]. The processing (adaptive segmentation, computing of parameters, cluster analysis) and the evaluation of the sleep record (using neural network) leads to the definition of a hypnogram.

\section{Results}

This method was tested on 8 sleep recordings (six children, two adults). The total time of all recordings was 70 hours. Success rate of this method is shown in Table 1 .

Success rate of the proposed method

Table 1

\begin{tabular}{|c|c|c|c|c|}
\hline Age & ID & Sex & Record length [h:m:s] & Success [\%] \\
\hline 6 & 3 & F & $10: 06: 30$ & 72.38 \\
\hline 8 & 5 & F & $09: 08: 30$ & 55.20 \\
\hline 8 & 6 & F & $07: 03: 30$ & 57.85 \\
\hline 9 & 4 & M & $08: 57: 00$ & 72.25 \\
\hline 11 & 1 & F & $07: 44: 00$ & 73.92 \\
\hline 15 & 2 & M & $09: 42: 30$ & 67.53 \\
\hline 47 & 7 & M & $08: 10: 00$ & 35.41 \\
\hline 47 & 8 & M & $08: 14: 00$ & 25.10 \\
\hline
\end{tabular}

The automatic sleep detection algorithm is primary intended for children's EEG records. We tested the method on adult's records too, but the success rate was minimal. The total success rate represents coincidence between two hypnograms. One hypnogram is created by a doctor and the second hypnogram is created using our algorithm. The success rate of sleep state detection is shown in Table 2.

The achieved success rate is a good starting point for further optimization of this method. More intensive cooperation with neurologists is needed to make the detection method more complete.
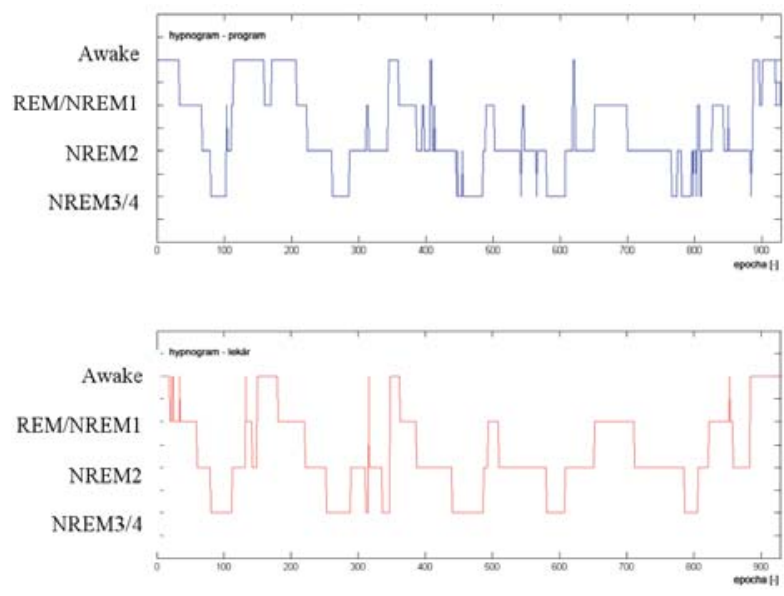

Fig. 4 Patient ID1
Success rate of the method based on sleep state detection Table 2

\begin{tabular}{|c|c|c|c|}
\hline \multicolumn{4}{|c|}{ Success rate of sleep state detection [\%] } \\
\hline NREM3/4 & NREM2 & REM/NREM1 & awake \\
\hline 78.00 & 81.39 & 55.21 & 56.04 \\
\hline
\end{tabular}

Results of all patients are displayed in Figs. 4-9. The blue line represents the hypnogram created by the proposed sleep detection algorithm and the red line represents the doctor-created hypnogram.

\section{Conclusion}

This paper deals with one of the possible methods for automatic sleep state detection in children. Only basic steps of this method are described in this paper. Correct identification of sleep states is a complex problem. Cooperation with a neurologist is of utmost importance in order to make this method more complete. The current detection success rate of awake and REM/NREM1 states is about $50 \%$, therefore in the first place a more complete detection of these states is necessary.

However, in view of the relatively high successful NREM3/4 state detection (78\%), these methods can be used for sleepwalking detection. Coincidently, one of tested patients was a somnambulant. In the video record the patient was awake, but the doctor detected NREM3/4 state and the same state was detected by the algorithm. Thus, in conjunction with a video recording, the proposed algorithm can also be a good tool for nurses, potentially alerting the nurse or doctor of a somnambulant patient during the course of sleep recording.
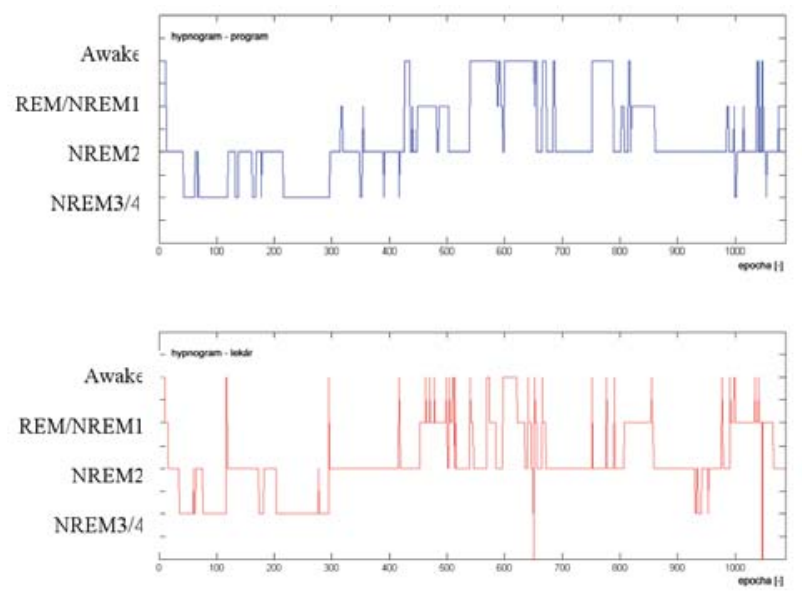

Fig. 5 Patient ID2 

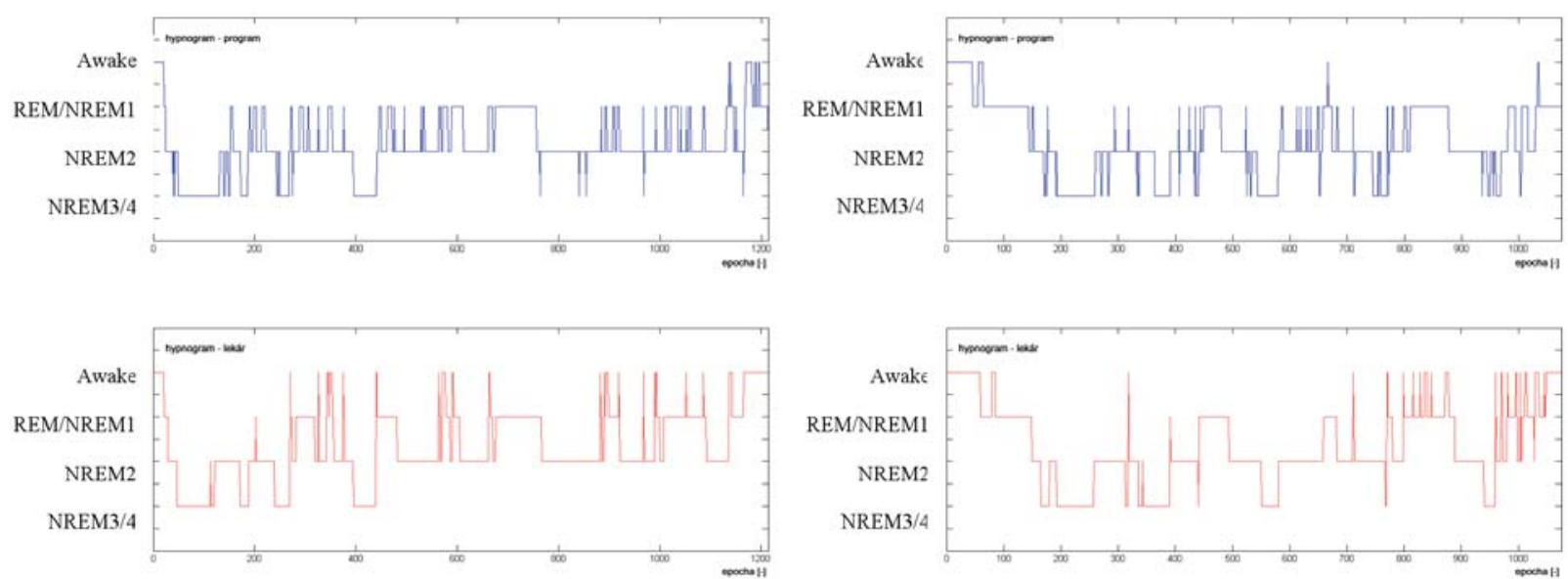

Fig. 6 Patient ID3

Fig. 7 Patient ID4
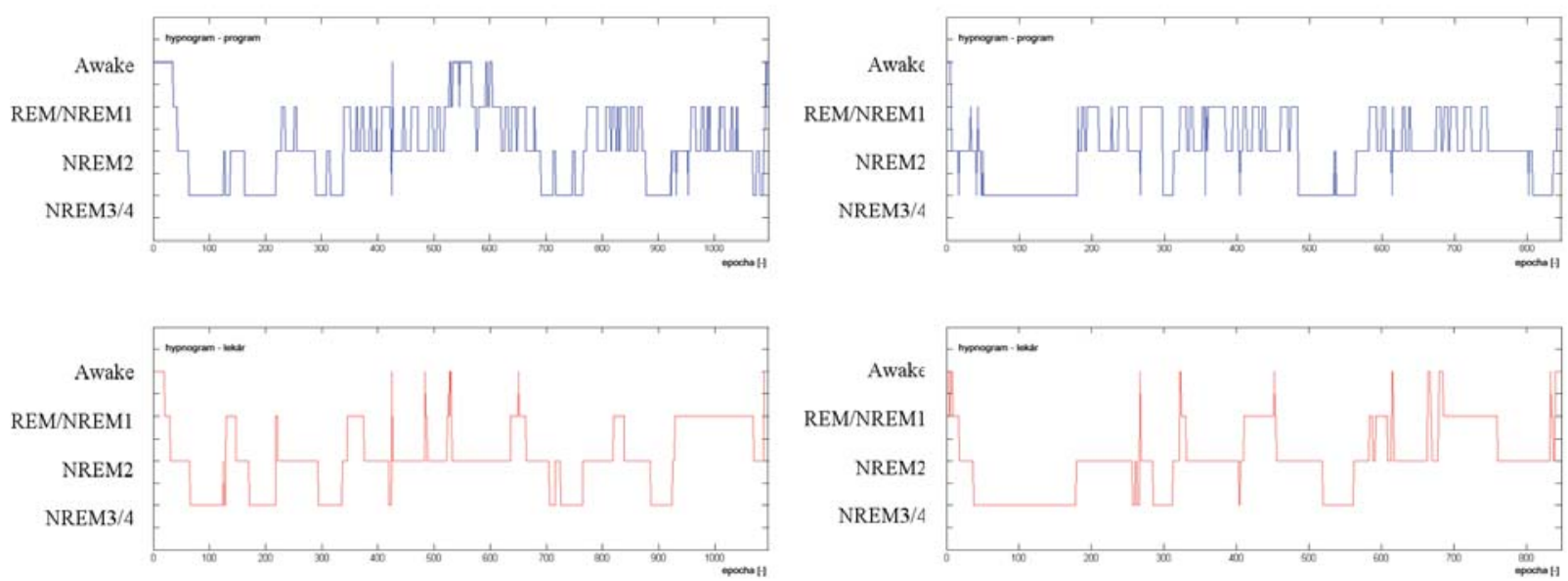

Fig. 8 Patient ID5

Fig. 9 Patient ID6

\section{References}

[1] IBER, C., ANCOLI, S., CHESSON, A: The AASM Manual of the Scoring of Sleep and Associated Events, American Academy of Sleep Medicine, Westchester, 2007.

[2] VONDRAK, I.: Artificial Intelligence and Neural Networks, FEI, VSB - TU : Ostrava, 2002.

[3] GALA M., MOHYLOVA, J.: Detection of K-Complex in the EEG Signal, www.springerlink.com:, Springer Berlin Heidelberg, 2010. Ed. O. Dossel, W C. Schlegel, Vol. 25, ISSN 1680-0737 (Print) 1433-9277 (Online); ISBN 978-3-642-03881-5 (Print) 978-3-64203882-2 (Online)

[4] MOHYLOVA, J., KRAJCA, V. Signal Processing in Medicine, ISBN 80-8070-341-8, 2005.

[5] HARTIGAN, J. A., WONG, M. A. A.: K-means Clustering Algorithm, Applied Statistics 28, 1979, pp. 100-108.

[6] KARDI, T.: K-mean Clustering Tutorials [online]. [2007-11-12]. WWW: 〈http://people.revoledu.com/kardi/tutorial/kMean/index.html> 